\title{
Adaptive Control of Nonlinear Teleoperation Systems with Varying Asymmetric Time Delays
}

\author{
Farzad Hashemzadeh ${ }^{\mathrm{a}, \mathrm{b}}$, Iraj Hassanzadeh ${ }^{\mathrm{a}, \mathrm{b}}$, Mahdi Tavakoli ${ }^{\mathrm{a}}$, Ghasem Alizadeh ${ }^{\mathrm{b}}$
}

\begin{abstract}
In this paper, a new adaptive control design method for nonlinear telerobotic systems with varying asymmetric time delays is presented. Using the proposed controller, it is possible to synchronize the state behavior of the local and the remote robots. While prior art on adaptive teleoperation has addressed stability in such systems only for constant delays, we guarantee asymptotic stability in the presence of delays that may be time-varying and/or unequal in the forward and backward directions. Using the proposed controller, asymptotic stability of the bilateral teleoperation system subject to any bounded varying delay with a bounded rate of variation can be guaranteed. The proposed controller also has the ability to cope with parameter variations in the dynamics of the local and the remote robots. To study the transparency of the closed-loop teleoperation system, we prove that the position and velocity errors between the local and the remote manipulators converge to zero asymptotically. To show the efficiency of the proposed controller, simulation results on a pair of two-degree-of-freedom manipulators with varying time delays in the communication channel are presented.
\end{abstract}

\section{INTRODUCTION}

Usion SING a telerobotic system, a human operator can carry out tasks in a remote environment. Different applications of telerobotic systems vary from tele-surgery to space manipulation. Teleoperation performance is greatly enhanced if haptic feedback about interaction occurring between the remote robot and the remote environment is provided to the human operator through the local robot [1]. Such systems are called "bilateral" because information flows in two directions between the operator and the remote environment [2]. On the other hand, in telerobotic applications with a distance between local and remote robots, there will be a time delay in the communication channel of the system [3]. The time delay in the closed-loop system can destabilize the telerobotic systems [3].

Control schemes have been developed to compensate for the time delay, most of which are based on the passivity

\footnotetext{
a Department of Electrical and Computer Engineering, University of Alberta, Edmonton, Alberta, T6G 2V4, Canada

${ }^{\mathrm{b}}$ Control Engineering Department, Faculty of Electrical and Computer Engineering, University of Tabriz, Tabriz, Iran.

E-mails: \{farzad.hashemzadeh, ihassanz, mahdi.tavakoli\}@ualberta.ca, alizadeh@tabrizu.ac.ir
}

theory. Passivity based control schemes [4] are inspired from energy interaction between interconnected systems [6]. To this end, Anderson and Spong [4] proposed scattering schemes based on the passivity theory. Another prominent passivity-based scheme is the wave variable formulation for a two port networks proposed by Neimeyer and Slotine [9]. These passivity based approaches can guarantee the passivity of bilateral teleoperation systems just for constant time delay and cannot preserve the passivity for varying time delays [7].

In most passivity based bilateral teleoperation architectures, only velocity and force information is transmitted between the local and the remote sides [8]. This means that only force and velocity tracking can be ensured in such architectures, leaving the possibility that any initial position mismatch between the local and the remote robots would lead to a position drift between the robots. To solve this problem, [9] transmit position information along with the velocity information through the communication channel. The Scattering and the wave variable approaches are the best known methods in the passivity approach, and have been the subject of recent studies concerning teleoperation under varying delays. An extension of the scattering approach to the case of varying time delays is reported in [5], in which a small positive gain is added in communication channel to dissipate the extra energy generated due to the distorted scattered signals caused by varying time delay. The gain should be less than $1-\dot{T}$, where $T$ is the instantaneous value of the varying time delay, such that communication channel remains passive. Also, an extended version of the wave variable approach with varying time delay was reported in [10], in which besides the wave variables, extra variables are transmitted in the communication channel to preserve passivity. Another interesting and recent method in passivity-based analysis of telerobotic systems is the synchronization-based approach [11]. Compared to the wave variable approach, in the synchronization-based methods power signals continue to be transmitted in the communication channel while the stability (rather than the passivity) of the overall telerobotic system is analyzed. The wave variable scheme, however, only analyzes the passivity of the communication channel in isolation, which is overly conservative, and in terms of performance suffers from unwanted wave reflection effects particularly for larger time delays. In synchronization-based schemes, all states including positions and velocities of local and remote robots act synchronously. The methods in adaptive synchronization-based schemes reported so far can only guarantee the stability for a constant time delay in the 
communication channel (this is not to be confused with considerable amount of previous work on non-adaptive state synchronization under varying time delay). In other words, in the presence of varying time delays, stability of the bilateral teleoperation systems cannot be preserved using the methods available so far.

In this paper, a new controlling scheme is proposed to guarantee stability of the bilateral teleoperation system and to synchronize the behavior of the local and the remote robots in the presence of varying time delays in communication channel. This paper is organized as follows. Section II concerns that telemanipulator dynamic model while the controller design method is presented in Section III. In Section IV, simulation results demonstrate the efficiency of the proposed method followed by the conclusion $\mathrm{n}$ presented in Section $\mathrm{V}$.

\section{TELE-MANIPULATOR DYNAMIC MODEL}

The local and the remote manipulators can be modeled by the following nonlinear equations:

$M_{l}\left(q_{l}\right) \ddot{q}_{l}+C_{l}\left(q_{l}, \dot{q}_{l}\right) \dot{q}_{l}+g_{l}\left(q_{l}\right)=\tau_{h}-\tau_{l}$

$M_{r}\left(q_{r}\right) \ddot{q}_{r}+C_{r}\left(q_{r}, \dot{q}_{r}\right) \dot{q}_{r}+g_{r}\left(q_{r}\right)=\tau_{r}-\tau_{e}$

where $q_{i}, \dot{q}_{i}$ and $\ddot{q}_{i}$ for $i \epsilon\{r, l\}$ are the joint positions, velocities and accelerations of the local and the remote robots, respectively. Also, $M_{i}\left(q_{i}\right), C_{i}\left(q_{i}, \dot{q}_{i}\right)$ and $g_{i}\left(q_{i}\right)$ are the inertia matrix, the Coriolis and centrifugal term and the gravitational force respectively, $\tau_{l}$ and $\tau_{r}$ are control torques for the local and the remote robots, and $\tau$ h and $\tau_{\mathrm{e}}$ are applied torques from the human operator and the environment sides respectively.

Some important properties of the above nonlinear dynamic model are $[12,13]$ :

For a manipulator with revolute joints, the inertia matrix $M_{i}\left(q_{i}\right)$ is symmetric positive definite and has the following upper and lower bounds:

$$
0<\lambda_{\min }\left(M_{i}\right) I \leq M_{i}\left(q_{i}\right) \leq \lambda_{\text {Max }}\left(M_{i}\right) I \leq \infty
$$

I. For a manipulator, the relation between the Coriolis/centrifugal and the inertia matrixes is as follows:

$$
\dot{M}_{i}\left(q_{i}\right)=C_{i}\left(q_{i}, \dot{q}_{i}\right)+C_{i}{ }^{T}\left(q_{i}, \dot{q}_{i}\right)
$$

II. For a manipulator with revolute joints, there exists a positive number $\eta$ bounding the Coriolis/centrifugal term as follows:

$$
\left|C_{i}\left(q_{i}, \dot{q}_{i}\right) \dot{q}_{i}\right| \leq \eta \dot{q}_{i}^{2}
$$

III. The nonlinear manipulator dynamics could be linearly parameterized as follows [13]:

$$
M_{i}\left(q_{i}\right) \ddot{q}_{i}+C_{i}\left(q_{i}, \dot{q}_{i}\right) \dot{q}_{i}+g_{i}\left(q_{i}\right)=Y_{i}\left(q_{i}, \dot{q}_{i}, \ddot{q}_{i}\right) \theta_{i}
$$

where $Y_{i}$ is a matrix of known functions of the generalized coordinates and their higher derivatives and $\theta_{\mathrm{i}}$ is a vector of the manipulator dynamic parameters.

\section{CONTROL Design}

In this part, the proposed controller design method to cope with varying time delays in a telerobotic system is presented. It is assumed that the dynamics of the system is not exact. So, the estimates of the robots' dynamics are employed in the controllers $\tau_{l}$ and $\tau_{r}$. The controllers $\tau_{1}$ and $\tau_{\mathrm{r}}$ in (1), are defined as follows

$$
\begin{aligned}
& \tau_{l}=-\widehat{M}_{l}\left(q_{l}\right) \dot{e}_{p l}-\hat{C}_{l}\left(q_{l}, \dot{q}_{l}\right) e_{p l}-\hat{g}_{l}\left(q_{l}\right)+\bar{\tau}_{l} \\
& \tau_{r}=\widehat{M}_{r}\left(q_{r}\right) \dot{e}_{p r}+\hat{C}_{r}\left(q_{r}, \dot{q}_{r}\right) e_{p r}+\hat{g}_{l}\left(q_{l}\right)-\bar{\tau}_{r}
\end{aligned}
$$

where ${ }^{\wedge}$ represents estimates of the remote and the local manipulators parameters and $\bar{\tau}_{i}$ for $i \epsilon\{l, r\}$ are the new control signals. Also, $e_{p l}$ and $e_{p r}$, which are position errors in local and remote sides, are defined as

$e_{p l} \triangleq q_{r}\left(t-T_{2}(t)\right)-q_{l}(t)$

$e_{p r} \triangleq q_{l}\left(t-T_{1}(t)\right)-q_{r}(t)$

where $T_{1}(t)$ is the delay in the feedforward path and $T_{2}(t)$ is the delay in the feedback path. The overall scheme of teleoperation with varying time delay is shown in Figure 1. We propose to define the new control signals, i.e., $\tau_{l}$ and $\tau_{r}$, as follows:

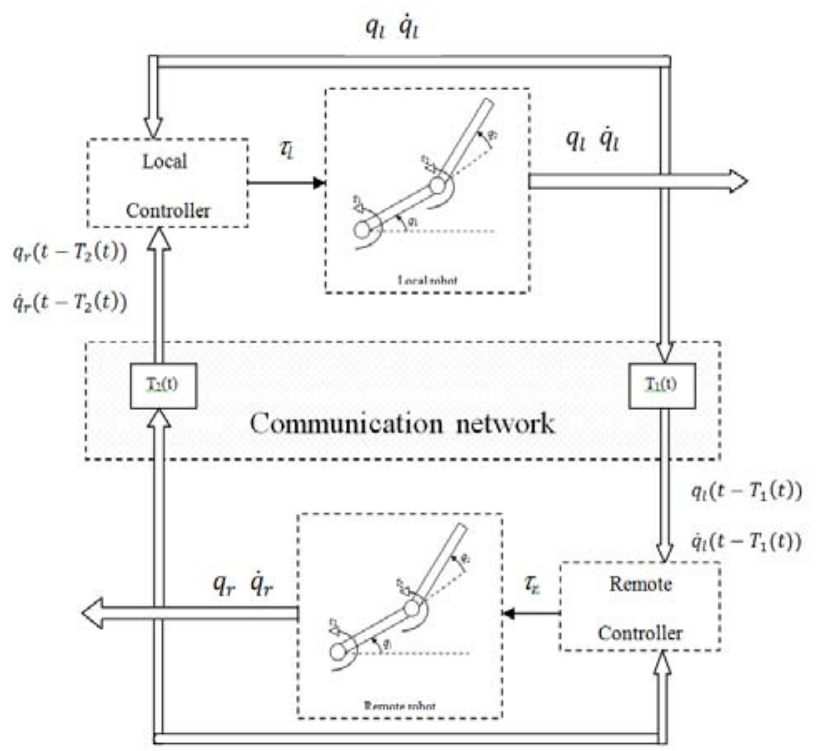

$q_{r} \dot{q}_{r}$

Fig. 1. The closed-loop teleoperation system under varying time delays

$\bar{\tau}_{r}=\left\{\begin{array}{cc}K_{r} \varepsilon_{r}-\frac{1}{2} \dot{e}_{p r}-\frac{1}{2} e_{v r}-\frac{e_{v r}^{T}\left(e_{p r}+\dot{e}_{p r}-e_{v r}\right)}{2\left\|\varepsilon_{r}\right\|_{2}^{2}} \varepsilon_{r}, & \left\|\varepsilon_{r}\right\|_{2} \neq 0 \\ 0 & \left\|\varepsilon_{r}\right\|_{2}=0\end{array}\right.$

$\bar{\tau}_{l}=\left\{\begin{array}{cc}K_{l} \varepsilon_{l}-\frac{1}{2} \dot{e}_{p l}-\frac{1}{2} e_{v l}-\frac{e_{v l}^{T}\left(e_{p l}+\dot{e}_{p l}-e_{v l}\right)}{2\left\|\varepsilon_{l}\right\|_{2}^{2}} \varepsilon_{l}, & \left\|\varepsilon_{l}\right\|_{2} \neq 0 \\ 0, & \left\|\varepsilon_{l}\right\|_{2}=0\end{array}\right.$

where $K_{i}$ for $i \epsilon\{l, r\}$ is a positive definite matrix and $\|\cdot\|_{2}$ denotes Euclidean norm. Also, $\dot{e}_{p i}$ is the time derivative of position error $e_{p i}$, and $e_{v i}$ (velocity error) and $\varepsilon_{i}$ for $i \epsilon\{l, r\}$ are defined as follows:

$\begin{array}{ll}\varepsilon_{i} \triangleq \dot{q}_{i}-e_{p i} & i \epsilon\{l, r\} \\ e_{v l} \triangleq \dot{q}_{r}\left(t-T_{2}(t)\right)-\dot{q}_{l} & \\ e_{v r} \triangleq \dot{q}_{l}\left(t-T_{1}(t)\right)-\dot{q}_{r} & \end{array}$

Note that because of the variation of time delays, the velocity error $e_{v i}$ and the derivative of the position error $\dot{e}_{p i}$ are not the same. Combining (2) and (1), the closed-loop system equations are found as

$M_{l}\left(q_{l}\right) \dot{\varepsilon}_{l}+C_{l}\left(q_{l}, \dot{q}_{l}\right) \varepsilon_{l}=-\widetilde{M}_{l}\left(q_{l}\right) \dot{e}_{p l}$

$-\tilde{C}_{l}\left(q_{l}, \dot{q}_{l}\right) e_{p l}-\tilde{g}_{l}\left(q_{l}\right)-\bar{\tau}_{l}+\tau_{h}$ 
$M_{r}\left(q_{r}\right) \dot{\varepsilon}_{r}+C_{r}\left(q_{r}, \dot{q}_{r}\right) \varepsilon_{r}=-\widetilde{M}_{r}\left(q_{r}\right) \dot{e}_{p r}-\tilde{C}_{r}\left(q_{r}, \dot{q}_{r}\right) e_{p r}-$ $\tilde{g}_{r}\left(q_{r}\right)-\bar{\tau}_{r}-\tau_{e}$

where $\sim$ represents the estimation error in the manipulator parameters., e.g., $\widetilde{M}_{i}=M_{i}-\widehat{M}_{i}, \tilde{C}_{i}=C_{i}-\hat{C}_{i}$, and $\tilde{g}_{i}=$ $g_{i}-\hat{\mathrm{g}}_{\mathrm{i}}$ for $\mathrm{i} \in\{\mathrm{l}, \mathrm{r}\}$. Using the fact that the equations of robot motions are linear in their parameters (Property IV), let us define the regressor matrix $Y_{\mathrm{i}}$ and the parameter vector $\theta$ such that the nominal robot dynamics can be written as

$M(q) \dot{e}+C(q, \dot{q}) e+g(q)=-Y(q, \dot{q}, e, \dot{e}) \theta$

Equation (7) can be achieved from Property IV via replacing $\ddot{q}$ with $\dot{e}$ and the exterior $\dot{q}$ in $C(q, \dot{q}) \dot{q}$ with $e$ and then negating $\mathrm{Y}$. Using the above linearity property, we have $Y_{i}\left(q_{i}, \dot{q}_{i}, e_{p i}, \dot{e}_{p i}\right) \tilde{\theta}_{i}=$

$-\widetilde{M}_{i}\left(q_{i}\right) \dot{e}_{p i}-\tilde{C}_{i}\left(q_{i}, \dot{q}_{i}\right) e_{p i}-\tilde{g}_{i}\left(q_{i}\right), \quad i \epsilon\{l, r\}$

where $\tilde{\theta}_{i}=\theta_{i}-\hat{\theta}_{i}$ and the regressor $Y_{i}\left(q_{i}, \dot{q}_{i}, e_{p i}, \dot{e}_{p i}\right)$ is a matrix whose elements are known functions of the generalized coordinates, derivatives of generalized coordinates, position errors and velocity errors. It is possible to find the following closed-loop dynamical equations from the above.

$M_{l}\left(q_{l}\right) \dot{\varepsilon}_{l}+C_{l}\left(q_{l}, \dot{q}_{l}\right) \varepsilon_{l}=$

$Y_{l}\left(q_{l}, \dot{q}_{l}, e_{p l}, \dot{e}_{p l}\right) \tilde{\theta}_{l}-\bar{\tau}_{l}+\tau_{h}$

$M_{r}\left(q_{r}\right) \dot{\varepsilon}_{r}+C_{r}\left(q_{r}, \dot{q}_{r}\right) \varepsilon_{r}=$

$Y_{r}\left(q_{r}, \dot{q}_{r}, e_{p r}, \dot{e}_{p r}\right) \tilde{\theta}_{r}-\bar{\tau}_{r}-\tau_{e}$

Now, we introduce the following adaptive update law for manipulators parameter estimation to be used in conjunction with the controllers (2):

$\dot{\hat{\theta}}_{i}=\Gamma Y_{i}^{T} \varepsilon_{i} \quad i \epsilon\{l, r\}$

In the following, we analyze the stability of the system.

Theorem I: In free motion $\left(\tau_{h}=\tau_{e}=0\right)$, the bilateral telemanipulator (1) with the controller (2)-(5) is asymptotically stable. Also, $q_{i}$ converges to a constant value, and the position error $e_{p i}$ and the velocity error $e_{v i}$ converge to zero for any bounded varying time delay with a bounded time derivative. Here, $i \epsilon\{l, r\}$.

Proof: To study the asymptotic stability under varying time delays in the communication channel, we use the following Lyapunov-Krasovskii functional:

$$
\begin{aligned}
V & =\frac{1}{2} \int_{t-T_{1}(t)}^{t} \dot{q}_{l}^{T} \dot{q}_{l} d t+\frac{1}{2} \int_{t-T_{2}(t)}^{t} \dot{q}_{r}{ }^{T} \dot{q}_{r} d t \\
& +\frac{1}{2} \sum_{i \in\{r, l\}}\left[\varepsilon_{i}^{T} M_{i} \varepsilon_{i}+\tilde{\theta}_{i}^{T} \Gamma^{-1} \tilde{\theta}_{i}+\frac{1}{2} e_{p i}{ }^{T} e_{p i}\right]
\end{aligned}
$$

where $\Gamma$ is a positive definite matrix. The time derivative of $V$ is

$\dot{V}=\sum_{i \in\{r, l\}}\left[\frac{1}{2} \varepsilon_{i}^{T} \dot{M} \varepsilon_{i}+\varepsilon_{i}{ }^{T} M \dot{\varepsilon}_{i}+\tilde{\theta}_{i}^{T} \Gamma^{-1} \dot{\tilde{\theta}}_{i}+\frac{1}{2} e_{p i}{ }^{T} \dot{e}_{p i}\right]$

$+\frac{1}{2} \dot{q}_{l}(t)^{T} \dot{q}_{l}(t)-\frac{1}{2}\left(1-\dot{T}_{1}\right) \dot{q}_{l}\left(t-T_{1}(t)\right)^{T} \dot{q}_{l}\left(t-T_{1}(t)\right)$

$+\frac{1}{2} \dot{q}_{r}(t)^{T} \dot{q}_{r}(t)-\frac{1}{2}\left(1-\dot{T}_{2}\right) \dot{q}_{r}\left(t-T_{2}(t)\right)^{T} \dot{q}_{r}(t$

Using equation (9), we can simplify $\dot{\mathrm{V}}$ as

$\dot{V}=\sum_{i \in\{r, l\}}\left[\frac{1}{2} \varepsilon_{i}{ }^{T} \dot{M} \varepsilon_{i}+\varepsilon_{i}{ }^{T}\left\{-C_{i} \varepsilon_{i}+Y_{i} \tilde{\theta}_{i}-\bar{\tau}_{i}\right\}+\tilde{\theta}_{i}{ }^{T} \Gamma^{-1} \dot{\tilde{\theta}}_{i}\right.$

$\left.+\frac{1}{2} e_{p i}^{T} \dot{e}_{p i}\right]+\frac{1}{2} \dot{q}_{l}(t)^{T} \dot{q}_{l}(t)$
$-\frac{1}{2}\left(1-\dot{\mathrm{T}}_{1}\right) \dot{\mathrm{q}}_{\mathrm{l}}\left(\mathrm{t}-\mathrm{T}_{1}(\mathrm{t})\right)^{\mathrm{T}} \dot{\mathrm{q}}_{\mathrm{l}}\left(\mathrm{t}-\mathrm{T}_{1}(\mathrm{t})\right)+\frac{1}{2} \dot{\mathrm{q}}_{\mathrm{r}}(\mathrm{t})^{\mathrm{T}} \dot{\mathrm{q}}_{\mathrm{r}}(\mathrm{t})$

$-\frac{1}{2}\left(1-\dot{\mathrm{T}}_{2}\right) \dot{\mathrm{q}}_{\mathrm{r}}\left(\mathrm{t}-\mathrm{T}_{2}(\mathrm{t})\right)^{\mathrm{T}} \dot{\mathrm{q}}_{\mathrm{r}}\left(\mathrm{t}-\mathrm{T}_{2}(\mathrm{t})\right)$

Using the following skew-symmetry property, which is equivalent to the property II,

$x^{T}\left(\dot{M}_{i}\left(q_{i}\right)-2 C_{i}\left(q_{i}, \dot{q}_{i}\right)\right) x=0 \quad \forall x \in R^{n}$

and after some simplifications, we get

$$
\begin{aligned}
& \frac{1}{2} \varepsilon_{i}{ }^{T} \dot{M} \varepsilon_{i}+\varepsilon_{i}{ }^{T}\left\{-C_{i} \varepsilon_{i}+Y_{i} \tilde{\theta}_{i}-\bar{\tau}_{i}\right\}+\tilde{\theta}_{i}{ }^{T} \Gamma^{-1} \dot{\tilde{\theta}}_{i} \\
& =\tilde{\theta}_{i}{ }^{T}\left\{Y_{i}^{T} \varepsilon_{i}+\Gamma^{-1} \dot{\tilde{\theta}}_{i}\right\}-\varepsilon_{i}{ }^{T} \bar{\tau}_{i}
\end{aligned}
$$

To simplify the right-hand side of (15), we introduce the following adaptive rule

$\dot{\tilde{\theta}}_{i}=-\Gamma Y_{i}^{T} \varepsilon_{i}$

With the assumption that the variation of unknown parameters $\theta$ is low, we get $\dot{\tilde{\theta}}=-\dot{\hat{\theta}}$, and the above adaptive rule for parameter updates becomes

$$
\dot{\hat{\theta}}_{i}=\Gamma Y_{i}^{T} \varepsilon_{i}
$$

which is same as (1). Using the above, it is possible to simplify $\dot{V}$, as follows:

$$
\begin{aligned}
& \dot{V}=\sum_{i \in\{r, l\}}\left[-\varepsilon_{i}{ }^{T} \bar{\tau}_{i}+\frac{1}{2} e_{p i}{ }^{T} \dot{e}_{p i}\right]+\frac{1}{2} \dot{q}_{l}(t)^{T} \dot{q}_{l}(t) \\
& -\frac{1}{2}\left(1-\dot{T}_{1}\right) \dot{q}_{l}\left(t-T_{1}(t)\right)^{T} \dot{q}_{l}\left(t-T_{1}(t)\right)+\frac{1}{2} \dot{q}_{r}(t)^{T} \dot{q}_{r}(t) \\
& -\frac{1}{2}\left(1-\dot{T}_{2}\right) \dot{q}_{r}\left(t-T_{2}(t)\right)^{T} \dot{q}_{r}\left(t-T_{2}(t)\right)
\end{aligned}
$$

Using the definition of $\bar{\tau}_{i}$ in (4) and after some manipulations, we get

$$
\begin{aligned}
& \dot{V}=\sum_{i \in\{r, l\}}\left[-\varepsilon_{i}{ }^{T} K_{i} \varepsilon_{i}+\frac{1}{2} \varepsilon_{i}{ }^{T} \dot{e}_{p i}+\frac{1}{2} \varepsilon_{i}{ }^{T} e_{v i}\right. \\
& \left.+\frac{1}{2} e_{v i}^{T}\left(e_{p i}+\dot{e}_{p i}-e_{v i}\right)+\frac{1}{2} e_{p i}{ }^{T} \dot{e}_{p i}\right] \\
& +\frac{1}{2} \dot{q}_{l}(t)^{T} \dot{q}_{l}(t)-\frac{1}{2}\left(1-\dot{T}_{1}\right) \dot{q}_{l}\left(t-T_{1}(t)\right)^{T} \dot{q}_{l}\left(t-T_{1}(t)\right) \\
& +\frac{1}{2} \dot{q}_{r}(t)^{T} \dot{q}_{r}(t)-\frac{1}{2}\left(1-\dot{T}_{2}\right) \dot{q}_{r}\left(t-T_{2}(t)\right)^{T} \dot{q}_{r}\left(t-T_{2}(t)\right)
\end{aligned}
$$

Applying the following relationships

$$
\begin{aligned}
& \frac{1}{2} \dot{q}_{l}(t)^{T} \dot{q}_{l}(t)-\frac{1}{2} \dot{q}_{r}\left(t-T_{2}(t)\right)^{T} \dot{q}_{r}\left(t-T_{2}(t)\right) \\
& =-\frac{1}{2} e_{v l}^{T} e_{v l}-\dot{q}_{l}(t)^{T} e_{v l}
\end{aligned}
$$

$$
\begin{aligned}
& \frac{1}{2} \dot{q}_{r}(t)^{T} \dot{q}_{r}(t)-\frac{1}{2} \dot{q}_{l}\left(t-T_{1}(t)\right)^{T} \dot{q}_{l}\left(t-T_{1}(t)\right) \\
& =-\frac{1}{2} e_{v r}^{T} e_{v r}-\dot{q}_{r}(t)^{T} e_{v r},
\end{aligned}
$$

it is found that

$$
\begin{aligned}
& \dot{V}=\sum_{i \epsilon\{r, l\}}\left[-\varepsilon_{i}{ }^{T} K_{i} \varepsilon_{i}+\frac{1}{2} \varepsilon_{i}{ }^{T} \dot{e}_{p i}+\frac{1}{2} \varepsilon_{i}^{T} e_{v i}\right. \\
& \left.+\frac{1}{2} e_{v i}^{T}\left(e_{p i}+\dot{e}_{p i}-e_{v i}\right)+\frac{1}{2} e_{p i}^{T} \dot{e}_{p i}\right]-\frac{1}{2} e_{v l}^{T} e_{v l}-\dot{q}_{l}(t)^{T} e_{v l} \\
& +\frac{1}{2} \dot{T}_{1} \dot{q}_{l}\left(t-T_{1}(t)\right)^{T} \dot{q}_{l}\left(t-T_{1}(t)\right)-\frac{1}{2} e_{v r}^{T} e_{v r}-\dot{q}_{r}(t)^{T} e_{v r} \\
& +\frac{1}{2} \dot{T}_{2} \dot{q}_{r}\left(t-T_{2}(t)\right)^{T} \dot{q}_{r}\left(t-T_{2}(t)\right)
\end{aligned}
$$

Considering the time derivatives of the position errors, $\dot{e}_{p l}$ and $\dot{e}_{p r}$ as 


$$
\begin{aligned}
& \frac{d\left(e_{p r}\right)}{d t}=\frac{d}{d t}\left(q_{l}\left(t-T_{1}(t)\right)-q_{r}(t)\right) \\
& =\left(1-\frac{d}{d t}\left(T_{1}(t)\right)\right) \dot{q}_{l}\left(t-T_{1}(t)\right)-\dot{q}_{r}(t) \\
& =\dot{q}_{l}\left(t-T_{1}(t)\right)-\dot{q}_{r}(t)-\frac{d}{d t}\left(T_{1}(t)\right) \dot{q}_{l}\left(t-T_{1}(t)\right) \\
& =e_{v r}-\dot{T}_{1} \dot{q}_{l}\left(t-T_{1}(t)\right) \\
& \frac{d\left(e_{p l}\right)}{d t}=\frac{d}{d t}\left(q_{r}\left(t-T_{2}(t)\right)-q_{l}(t)\right) \\
& =\left(1-\frac{d}{d t}\left(T_{2}(t)\right)\right) \dot{q}_{r}\left(t-T_{2}(t)\right)-\dot{q}_{l}(t) \\
& =\dot{q}_{r}\left(t-T_{2}(t)\right)-\dot{q}_{l}(t)-\frac{d}{d t}\left(T_{2}(t)\right) \dot{q}_{r}\left(t-T_{2}(t)\right)=e_{v l}- \\
& \dot{T}_{2} \dot{q}_{r}\left(t-T_{2}(t)\right),
\end{aligned}
$$

we get the following relationships between $\dot{e}_{p i}$ and $e_{v i}$ :

$$
\dot{e}_{p l}=e_{v l}-\dot{T}_{2} \dot{q}_{r}\left(t-T_{2}(t)\right)
$$

$$
\dot{e}_{p r}=e_{v r}-\dot{T}_{1} \dot{q}_{l}\left(t-T_{1}(t)\right)
$$

Applying (24)-(25) to (22), $\dot{V}$ could be simplified as becomes

$$
\begin{gathered}
\dot{V}=\sum_{i \in\{r, l\}}\left[-\varepsilon_{i}^{T} K_{i} \varepsilon_{i}+\frac{1}{2} \varepsilon_{i}^{T} \dot{e}_{p i}+\frac{1}{2} \varepsilon_{i}^{T} e_{v i}\right. \\
\left.+\frac{1}{2} e_{v i}^{T}\left(e_{p i}+\dot{e}_{p i}-e_{v i}\right)+\frac{1}{2} e_{p i}{ }^{T} \dot{e}_{p i}-\frac{1}{2} e_{v i}^{T} e_{v i}-\dot{q}_{i}(t)^{T} e_{v i}\right] \\
+\frac{1}{2} \dot{q}_{l}\left(t-T_{1}(t)\right)^{T}\left(e_{v r}-\dot{e}_{p r}\right) \\
+\frac{1}{2} \dot{q}_{r}\left(t-T_{2}(t)\right)^{T}\left(e_{v l}-\dot{e}_{p l}\right)
\end{gathered}
$$

Considering that

$$
\dot{q}_{l}\left(t-T_{1}(t)\right)=e_{v r}+\dot{q}_{r}
$$

$\dot{q}_{r}\left(t-T_{2}(t)\right)=e_{v l}+\dot{q}_{l}$

(28)

$\dot{V}$ is further simplified to

$\dot{V}=\sum_{i \in\{r, l\}}\left[-\varepsilon_{i}^{T} K_{i} \varepsilon_{i}+\frac{1}{2} \varepsilon_{i}^{T} \dot{e}_{p i}+\frac{1}{2} \varepsilon_{i}^{T} e_{v i}\right.$

$+\frac{1}{2} e_{v i}^{T}\left(e_{p i}+\dot{e}_{p i}-e_{v i}\right)+\frac{1}{2} e_{p i}{ }^{T} \dot{e}_{p i}-\frac{1}{2} e_{v i}^{T} e_{v i}-\dot{q}_{i}(t)^{T} e_{v i}$

$\left.+\frac{1}{2}\left(e_{v i}+\dot{q}_{i}\right)^{T}\left(e_{v i}-\dot{e}_{p i}\right)\right]$

More simplification gives

$\dot{V}=\sum_{i \in\{r, l\}}\left[-\varepsilon_{i}^{T} K_{i} \varepsilon_{i}-\frac{1}{2} e_{v i}^{T} e_{v i}+\frac{1}{2}\left\{\varepsilon_{i}-\dot{q}_{i}(t)+e_{p i}\right\}^{T} \dot{e}_{p i}\right.$

$\left.+\frac{1}{2}\left\{\varepsilon_{i}-\dot{q}_{i}(t)+e_{p i}\right\}^{T} e_{v i}\right]$

(30)

Using the definition of $\varepsilon_{i}$ in (5), negative semidefiniteness of $\dot{V}$ is seen as

$\dot{V}=-\sum_{i \epsilon\{r, l\}}\left[\varepsilon_{i}{ }^{T} K_{i} \varepsilon_{i}+\frac{1}{2} e_{v i}{ }^{T} e_{v i}\right]$

Integrating (31), it is easy to see that $V(t)-V(0)=$ $\int_{o}^{t} \dot{V}=-\left(\int_{o}^{t}\left(\varepsilon_{i}{ }^{T} K_{i} \varepsilon_{i}+\frac{1}{2} e_{v i}{ }^{T} e_{v i}\right)\right) \leq 0$, i.e., $V(t) \leq V(0)$. Using the fact that $V(t) \geq 0, V(t) \leq V(0)$ and $\dot{V}(t) \leq 0$, it is possible to say that $V(t)$ is positive bounded decreasing function. Thus, it is concluded that all terms in $V(t)$ are bounded. Now let us proceed to the analysis of transparency of the system by proving $\lim _{t \rightarrow \infty} e_{v i}(t)=\lim _{t \rightarrow \infty} e_{p i}(t)$ $=0$. We will also show that $\lim _{t \rightarrow \infty} q_{i}(t)$ is bounded to establish closed-loop stability. Previously, it is shown that $V(t)$ is bounded, so all terms in $V(t)$ including $\varepsilon_{i}, \dot{q}_{i} e_{i}$ and $\tilde{\theta}_{i} \in \mathcal{L}_{\infty}$. Using $V(t) \geq 0$ and integrating equation (31), $\int_{o}^{t}\left(\varepsilon_{i}{ }^{T} K_{i} \varepsilon_{i}\right)+\frac{1}{2} \int_{o}^{t}\left(e_{v i}{ }^{T} e_{v i}\right)=V(0)-V(t) \leq V(0), \quad$ it follows that $\varepsilon_{i}, e_{v i} \in \mathcal{L}_{2}$. It is easy to see from (5) that, since $\dot{q}_{i} \in \mathcal{L}_{\infty}$, we have $e_{v i} \in \mathcal{L}_{\infty}$. Combining these with (24)-(25) and the assumption that $\dot{T}_{i}$ is bounded, it is seen that $\dot{e}_{p i} \in \mathcal{L}_{\infty}$. All these bounded signals result in the boundedness of the regressor matrix $Y_{i}$, i.e., $Y_{i} \in \mathcal{L}_{\infty}$. Using the boundedness of $\varepsilon_{i}, \tilde{\theta}_{i}, \bar{\tau}_{i}$ and $Y_{i}$ and Properties I and III in (9), it is seen that $\dot{\varepsilon}_{i} \in \mathcal{L}_{\infty}$. Using Barbalat's lemma, given that $\varepsilon_{i} \in \mathcal{L}_{2}$ and $\dot{\varepsilon}_{i} \in \mathcal{L}_{\infty}$ , it is concluded that $\lim _{t \rightarrow \infty} \varepsilon_{i}=0$. Using $\dot{\varepsilon}_{i}=\ddot{q}_{i}-\dot{e}_{p i}$, it is determined that $\ddot{q} \in \mathcal{L}_{\infty}$. Invoking the time derivative of $e_{v i}$, e.g., $\dot{e}_{v l}=\left(1-\dot{T}_{2}\right) \ddot{q}_{r}\left(t-T_{2}\right)-\ddot{q}_{l}$, it is concluded that $\dot{e}_{v i} \in \mathcal{L}_{\infty}$. Therefore, using Barbalat's lemma again, since $e_{v i} \in \mathcal{L}_{2}$ and $\dot{e}_{v i} \in \mathcal{L}_{\infty}$, it is resulted that $\lim _{t \rightarrow \infty} e_{v i}=0$. Replacing $e_{p l}=q_{r}\left(t-T_{2}(t)\right)-q_{l}(t)$ in $\varepsilon_{l}=\dot{q}_{l}-e_{p l}$ and using the fact that $\varepsilon_{l} \rightarrow 0$, stability of the system $\dot{q}_{l}(t)+$ $q_{l}(t)=q_{r}\left(t-T_{2}(t)\right)$ can be analyzed with calculating the response of $q_{l}(t)$ to the $q_{r}\left(t-T_{2}(t)\right)$. Homogenous response of stable differential equation $\dot{q}_{l}(t)+q_{l}(t)=$ $q_{r}\left(t-T_{2}(t)\right)$ is $q_{l}(t)=e^{-t} \int e^{t} q_{r}\left(t-T_{2}(t)\right)$. Similar result could be achieved for $q_{r}(t)$ as $q_{r}(t)=e^{-t} \int e^{t} q_{l}(t-$ $\left.T_{1}(t)\right)$. If $\int e^{t} q_{r}\left(t-T_{2}(t)\right)$ is bounded then $q_{l} \rightarrow 0$, which implies that $q_{r} \rightarrow 0, e_{p r}$ and $e_{p l} \rightarrow 0$. If $\int e^{t} q_{r}\left(t-T_{2}(t)\right)$ be unbounded then $q_{l}(t)$ would be indeterminate which could be evaluated using Hopital's rule as $\lim _{t \rightarrow \infty} q_{r}(t)=$ $\frac{\frac{d}{d t}\left(\int e^{t} q_{l}\left(t-T_{1}(t)\right)\right)}{\frac{d}{d t}\left(e^{t}\right)}=q_{l}\left(t-T_{1}(t)\right)$, i.e., $e_{p r} \rightarrow 0$. Similar results could be achieved for $e_{p l} \rightarrow 0$. Using the definition of $\varepsilon_{i}$ in (5) and using the fact that $\lim _{t \rightarrow \infty} \varepsilon_{i}(t)=0$ and $\lim _{t \rightarrow \infty} e_{p i}(t)=0$, it is easy to see from (5) that $\lim _{t \rightarrow \infty} \dot{q}_{i}(t)=0$ and, in other words, $\lim _{t \rightarrow \infty} q_{i}(t)=$ Constant. Thus, it was proved that $\lim _{t \rightarrow \infty} q_{i}(t)=$ Constant and $\lim _{t \rightarrow \infty} e_{v i}=\lim _{t \rightarrow \infty} e_{p i}=0 \quad i \epsilon\{l, r\}$

Thus, in free motion of the bilateral telemanipulation system (1), state synchronization is satisfied under time varying communication delays. Also, the closed-loop telemanipulator is input-to-state stable from the human and environment input forces to the local and remote manipulator states.

\section{Simulation Results}

To verify the theoretical results of this paper, the local and remote manipulators are considered to be a pair of twodegree-of-freedom serial robots with revolute joints. The local and remote manipulator dynamics (1) have the following elements of inertia, Coriolis/centrifugal and gravity matrixes: 
$M_{i}\left(q_{i}\right)=\left[\begin{array}{ll}M_{i_{11}} & M_{i_{12}} \\ M_{i_{21}} & M_{i_{22}}\end{array}\right], \quad C_{i}\left(q_{i}, \dot{q}_{i}\right)=\left[\begin{array}{ll}C_{i_{11}} & C_{i_{12}} \\ C_{i_{21}} & C_{i_{22}}\end{array}\right]$ and $g_{i}\left(q_{i}\right)=\left[\begin{array}{l}g_{i_{1}} \\ g_{i_{2}}\end{array}\right] \quad$ where for $i \in\{l, r\} \quad, \quad M_{i_{11}}=l_{i_{2}}^{2} m_{i_{2}}+$ $l_{i_{1}}^{2}\left(m_{i_{1}}+m_{i_{2}}\right)+2 l_{i_{1}} l_{i_{2}} m_{i_{2}} \cos \left(q_{i_{2}}\right), \quad M_{i_{12}}=M_{i_{21}}=l_{i_{2}}^{2} m_{i_{2}}+$ $l_{i_{1}} l_{i_{2}} m_{i_{2}} \cos \left(q_{i_{2}}\right), M_{i_{22}}=l_{i_{2}}^{2} m_{i_{2}}, C_{i_{11}}=-2 l_{i_{1}} l_{i_{2}} m_{i_{2}} \sin \left(q_{i_{2}}\right) \dot{q}_{i_{2}}$, $C_{i_{12}}=-l_{i_{1}} l_{i_{2}} m_{i_{2}} \sin \left(q_{i_{2}}\right) \dot{q}_{i_{2}}, C_{i_{21}}=l_{i_{1}} l_{i_{2}} m_{i_{2}} \sin \left(q_{i_{2}}\right) \dot{q}_{i_{1}}, C_{i_{22}}=$ $0, \quad g i_{1}=g l_{i_{2}} m_{i_{2}} \cos \left(q_{i_{1}}+q_{i_{2}}\right)+l_{i_{1}}\left(m_{i_{1}}+m_{i_{2}}\right) \quad \cos \left(q_{i_{1}}\right)$, $g_{i_{2}}=g l_{i_{2}} m_{i_{2}} \cos \left(q_{i_{1}}+q_{i_{2}}\right)$. Here, $q_{i_{1}}$ and $q_{i_{2}}$ are the positions of the first and the second revolute joints, $l_{i_{1}}$ and $l_{i_{2}}$ are the link lengths and $m_{i_{1}}$ and $m_{i_{2}}$ are the masses of the first and the second links for each robot. For both manipulators, we used the same linear parameterization (see Property IV) as in [11]:

$Y_{i}\left(q_{i}, \dot{q}_{i}, e_{p i}, \dot{e}_{p i}\right)=\left[\begin{array}{lllll}Y_{i_{11}} & Y_{i_{12}} & Y_{i_{13}} & Y_{i_{14}} & Y_{i_{15}} \\ Y_{i_{21}} & Y_{i_{22}} & Y_{i_{23}} & Y_{i_{24}} & Y_{i_{25}}\end{array}\right] \quad, \quad \hat{\theta}_{i}=$ $\left[\begin{array}{lllll}\hat{\theta}_{i_{1}} & \hat{\theta}_{i_{2}} & \hat{\theta}_{i_{3}} & \hat{\theta}_{i_{4}} & \hat{\theta}_{i_{5}}\end{array}\right]$

where, $Y_{i_{11}}=-\dot{e}_{p i_{1}}, Y_{i_{12}}=-2 \dot{e}_{p i_{1}} \cos \left(q_{i_{2}}\right)-\dot{e}_{p i_{2}} \cos \left(q_{i_{2}}\right)+$ $\dot{q}_{i_{2}} e_{p i_{2}} \sin \left(q_{i_{2}}\right)+2 e_{p i_{1}} \dot{q}_{i_{2}} \sin \left(q_{i_{2}}\right) \quad, \quad Y_{i_{13}}=-\dot{e}_{p i_{2}} \quad, \quad Y_{i_{14}}=$ $-g \cos \left(q_{i_{1}}+q_{i_{2}}\right) \quad, \quad Y_{i_{15}}=-g \cos \left(q_{i_{1}}\right), \quad Y_{i_{21}}=0 \quad, \quad Y_{i_{22}}=$ $-\dot{e}_{p i_{1}} \cos \left(q_{i_{2}}\right)-\dot{q}_{i_{1}} e_{p i_{1}} \sin \left(q_{i_{2}}\right), \quad Y_{i_{23}}=-\dot{e}_{p i_{1}}-\dot{e}_{p i_{2}}, \quad Y_{i_{24}}=$ $-g \cos \left(q_{i_{1}}+q_{i_{2}}\right), Y_{i_{25}}=0$ and $\hat{\theta}_{i_{1}}=\hat{l}_{i_{2}}^{2} \widehat{m}_{i_{2}}+\hat{l}_{i_{1}}^{2}\left(\widehat{m}_{i_{1}}+\widehat{m}_{i_{2}}\right)$, $\hat{\theta}_{i_{2}}=\hat{l}_{i_{1}} \hat{l}_{i_{2}} \widehat{m}_{i_{2}}, \hat{\theta}_{i_{3}}=\hat{l}_{i_{2}}^{2} \widehat{m}_{i_{2}}, \hat{\theta}_{i_{4}}=\hat{l}_{i_{2}} \widehat{m}_{i_{2}}, \hat{\theta}_{i_{5}}=\hat{l}_{i_{1}}\left(\widehat{m}_{i_{1}}+\right.$ $m i 2, i \epsilon l, r$.

Using the above definitions for the elements of the matrix $\hat{\theta}_{i}$, it is possible to estimate matrixes $M_{i}, C_{i}$ and $g_{i}$ based on the elements of $\hat{\theta}_{i}$ that will be estimated online. In simulations, the physical parameters of the manipulators are set to $m_{l_{1}}=4 \mathrm{~kg}, m_{l_{2}}=0.5 \mathrm{~kg}, l_{l_{1}}=50 \mathrm{~cm}, l_{l_{2}}=50 \mathrm{~cm}$, $m_{r_{1}}=3.4 \mathrm{~kg}, m_{r_{2}}=0.25 \mathrm{~kg}, l_{r_{1}}=50 \mathrm{~cm}, l_{r_{2}}=50 \mathrm{~cm}$ and the controller gain $K_{i}$ is set to $3 I$. In the following, three simulation scenarios are considered involving constant time delays, random time delays and sinusoidal time delays (scenarios A, B and C, respectively). A human torque, which is shown in Figure 2, is applied to the local manipulator and the tracking performance of the first and the second joints of the local and remote manipulators are considered.

\section{A) Simulation with constant time delays}

In Figure 3, simulation results for a constant time delay similar to that used in [11], $T_{1}=0.4$ and $T_{2}=0.4$ seconds, in terms of joint positions of the remote manipulator and delayed joint positions of the local manipulator in the presences of the exerted human torque are shown. Comparing the results, it can be seen that the results of the proposed method is exactly the same as that of the method in [11]. This similarity is because of the fact that in this simulation time delays are constant.

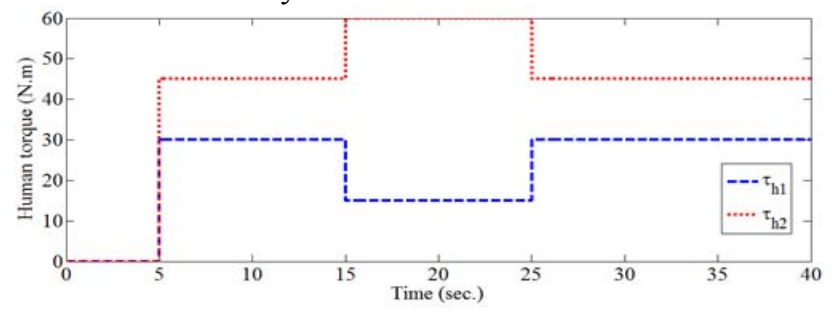

Fig. 2. Exerted human torque

\section{B) Simulation with random time delays}

In this part, simulation results of the proposed method compared with [11] for random time delays with Gaussian distribution with mean 0.48 second and standard deviation of 0.022 , are shown. In this simulation, again the positions of the first and the second joints of the remote manipulator compared with delayed joint positions of the local manipulator in the presences of exerted human torque are shown. As it can be seen from Figure 4, the proposed method has better tracking performance and less fluctuations and settling time than the method in [11].

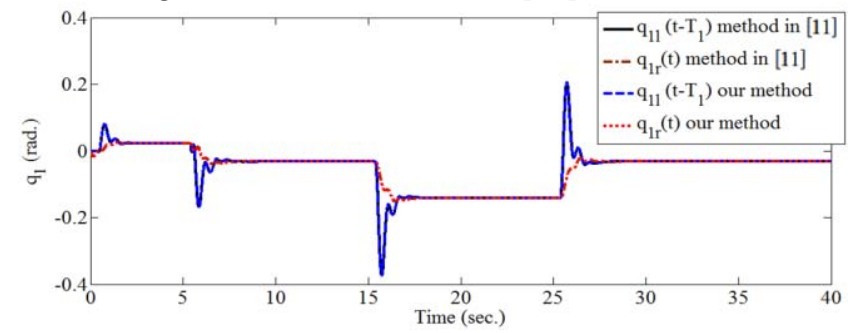

Fig. 3a. Positions of the first joints of the local and remote manipulator in telemanipulation with constant time delay.

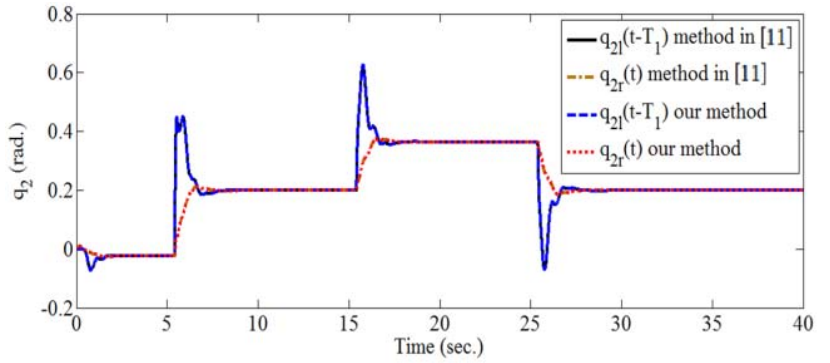

Fig. 3b. Positions of the second joints of the local and remote manipulator in telemanipulation with constant time delay

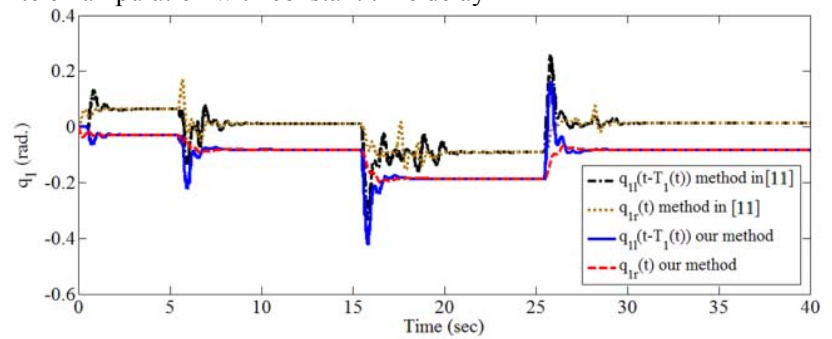

Fig. 4a. Positions of the first joints of the local and remote manipulators in telemanipulation with random time delay

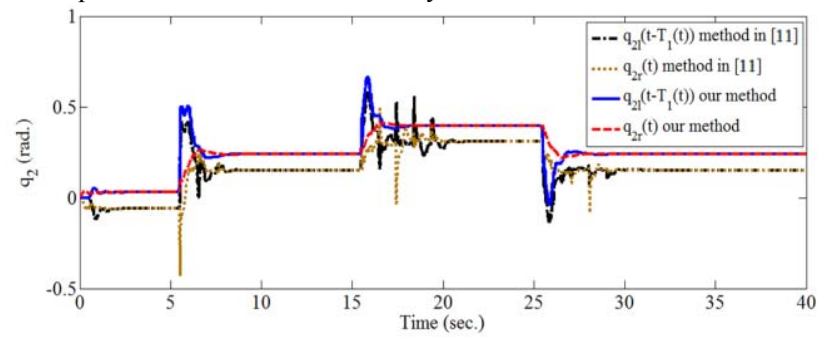

Fig. 4b. Positions of the second joints of the local and remote manipulators in telemanipulation with random time delay

\section{C) Simulation with sinusoidal time delays}

Let us verify the telemanipulator's free motion tracking performance under sinusoidal time varying delays. The feedforward and feedback delays in the communication channel are assumed to be changing as sinusoids with a mean of 1 second and frequencies of 0.5027 and 0.4714 
$\mathrm{rad} / \mathrm{sec}-$ see Figure 5. Joint positions of the local and the remote manipulators in the presences of the exerted human torque of Figure 2 are shown in Figure 6. It is remarkable that state synchronization of the bilateral teleoperation system is satisfied in the presence of the fast varying communication delays. In Figure 7 , tracking errors in the first and the second joints of the local and remote manipulators are shown, which are asymptotically converging to zero as predicted by the theory.

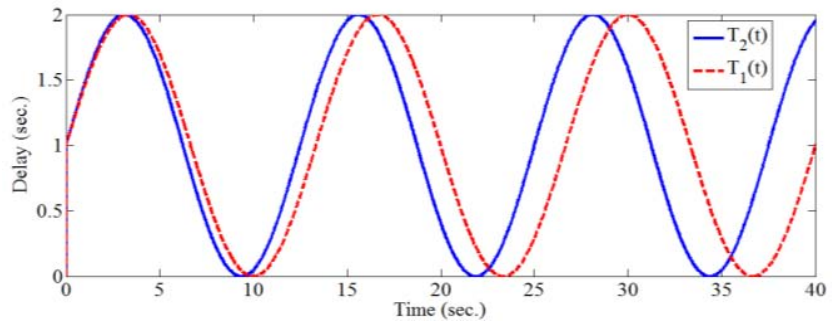

Fig. 5. Time varying delays in communication channel

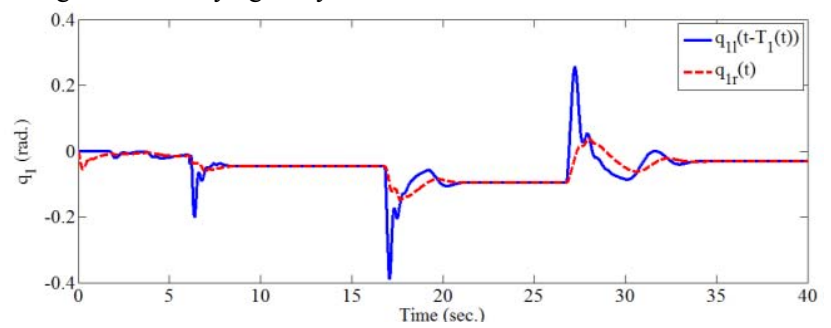

Fig. 6a. Positions of the first joints of the local and remote manipulators in telemanipulation with sinusoidal time varying delay

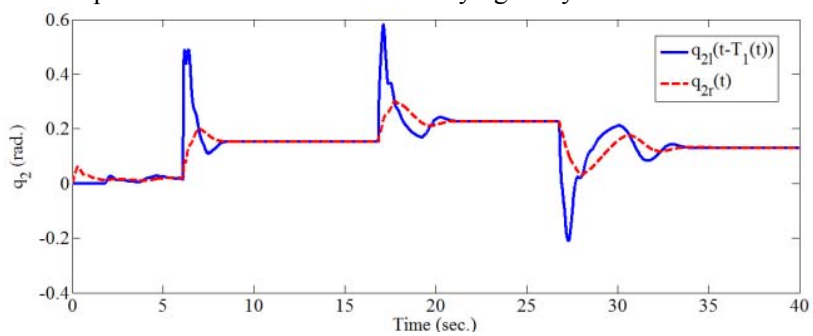

Fig. 6b. Positions of the second joints of the local and remote manipulators in telemanipulation with sinusidal time varying delay

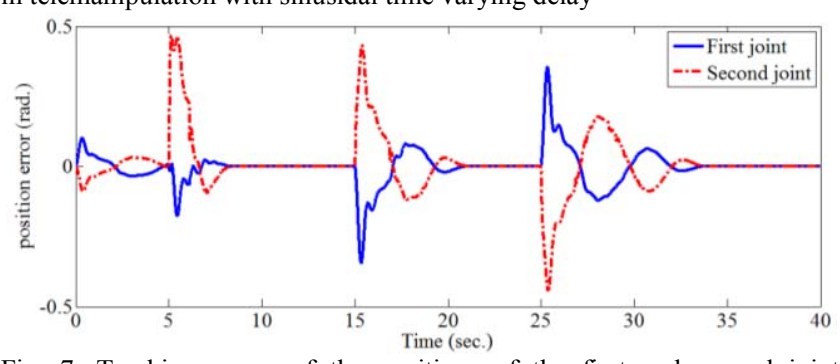

Fig. 7. Tracking errors of the positions of the first and second joints between the local and remote manipulators

\section{V.CONCLUSION AND FUTURE WORKS}

In this paper a new state synchronizing controller for bilateral teleoperation systems with varying time delays in the communication channel is proposed. Lyapunov stability of the closed-loop system in the presence of time varying delays is established. Besides, it is proved and also shown via simulations that, using the proposed controller, asymptotic synchronization between the local and the remote robots occurs. The proposed controller entails an adaptive tuning rule in the local and remote sides to estimate the unknown/uncertain dynamic parameters of the manipulators. Thus, in this paper, only the estimated values of the robots' parameters are used in the controller, when providing the asymptotic state synchronization between the local and the remote robots under varying time delays. As future work, state synchronization under varying time delays in with hard-contact telemanipulation with consideration for force tracking to obtain full transparency will be studied.

\section{REFERENCES}

[1] C.-P. Kuan and K. Young, "VR-Based Teleoperation for Robots Compliance Control," Journal of Intelligent \& Robotic Systems, vol. 30, no. 4, pp. 377-398, 2001.

[2] H. Baier and G. Schmidt, "Transparency and Stability of Bilateral Kinesthetic Teleoperation with Time-Delayed Communication," Journal of Intelligent \& Robotic Systems, vol. 40, No. 1, pp. 1-22, 2004.

[3] T. B. Sheridan, "Space teleoperation through time delay: Review and prognosis," IEEE Transactions on Robotics and Automation, vol. 9, no. 5, pp. 592-606, 1993.

[4] R.J. Anderson and M.W. Spong, "Asymptotic stability for force reflecting teleoperations with time delay," IEEE International Conference on Robotics and Automation, pp. 1618-1625, 1989.

[5] R. Lozano, N. Chopra, and M.W. Spong, "Passivation of force reflecting bilateral teleoperators with time varying delay," In Proceedings of the 8th Mechatronics Forum, pp. 954-962, 2002.

[6] C.A. Desoer and M. Vidyasagar, "Feedback systems: Input-output properties," New York: Academic Press, 1975.

[7] P.F. Hokayem and M.W. Spong, "Bilateral teleoperation: An historical survey," Automatica, vol. 42 (12), pp. 2035-2057, 2006.

[8] K. Hosseini-Suny, H. Momeni, and F. Janabi-Sharifi, "Model Reference Adaptive Control Design for a Teleoperation Systems with Output Prediction," Journal of Intelligent \& Robotic Systems, vol. 59, no. 3-4, pp. 319-339, 2010.

[9] N. Chopra and M.W. Spong, "Passivity-based control of multi-agent systems," Advances in robot control: from everyday physics to human-like movements, pp. 107-134, Springer, Berlin, 2006.

[10] Y. Gu, C. Zhang, K.T. Chong, "Adaptive passive control with varying time delay," Simulation Modelling Practice and Theory, vol. 18 (1), pp. 1-8, 2010.

[11] E. Nuño, R. Ortega, and L. Basañez, "An adaptive controller for nonlinear teleoperators," Automatica, vol. 46 (1), pp. 155-159, 2010.

[12] R. Kelly, V. Santibáñez, and A. Loria, "Control of robot manipulators in joint space," Springer, Berlin 2005.

[13] M. W. Spong, S. Hutchinson, and M. Vidyasagar, "Robot modeling and control," Wiley, 2006. 